\title{
PRINCIPAL LINE BASED ICP ALIGNMENT FOR PALMPRINT VERIFICATION
}

\author{
Wei $\mathrm{Li}^{1}$, Lei Zhang ${ }^{2}$, David Zhang ${ }^{2}$ and Jingqi Yan ${ }^{1}$ \\ ${ }^{1}$ Institute of Image Processing and Pattern Recognition, Shanghai Jiao Tong University, Shanghai, \\ China \\ ${ }^{2}$ Biometrics Research Center, Dept. of Computing, The Hong Kong Polytechnic University, Hong Kong
}

\begin{abstract}
Image alignment is a crucial step in palmprint verification. However, most of the existing palmprint alignment methods use only some key points between fingers or in palm boundary to extract the region of interest (ROI), which is consequently used for feature extraction and matching. Such alignment methods can only give a coarse alignment of the palmprint images. This paper presents a new effective refinement method for palmprint alignment by adapting the iterative closest point (ICP) method to the palmprint principal lines. The proposed method offers a more accurate alignment of palmprints by correcting efficiently the shifting, rotation and scaling variations introduced in data acquisition. The experimental results show that the proposed method can greatly improve the palmprint verification accuracy in real time.
\end{abstract}

Index Terms - Palmprint recognition, image alignment, line extraction, ICP, biometrics

\section{INTRODUCTION}

Automatic personal authentication using biometric information is playing an important role in applications of public security, access control, forensic, e-banking, etc. Many kinds of biometric authentication techniques have been developed based on different biometric characteristics, including physiological-based (such as fingerprint, face, iris, palmprint, hand shape, etc.) and behavioral-based (such as signature, voice, gait, etc.) characteristics. Palmprint has been widely studied in the past decade and it has proven to be a unique biometric identifier. Palmprint systems have merits of high accuracy, low cost, user friendliness, etc.

Various palmprint verification systems have been set up and many algorithms have been proposed [1-8]. The main processes of palmprint verification include data collection, region of interest (ROI) extraction, feature extraction, matching and decision making. ROI extraction is a very important step which affects greatly the following steps. It aligns the palmprints and normalizes the area for feature extraction and matching. Most of the existing methods extract ROI according to some key points between fingers or in palm boundary [1-3], as shown in Fig. 1.

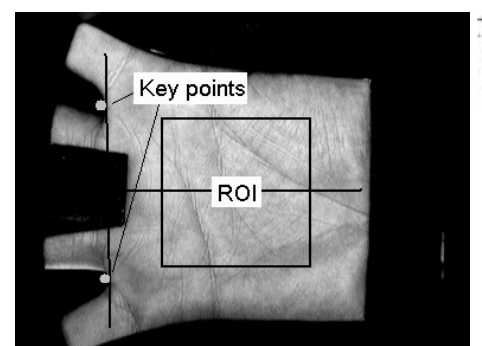

(a)

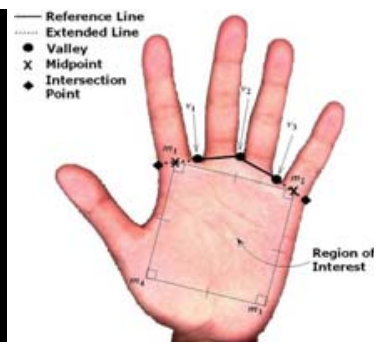

(b)
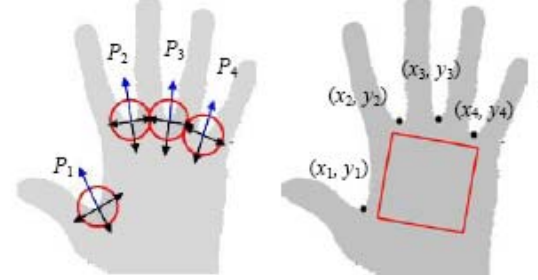

(c)

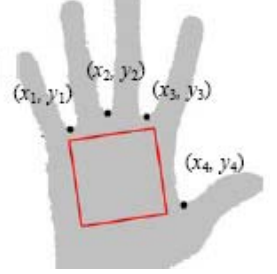

Figure 1. Illustration of three typical ROI extraction methods by using (a) two key points [1]; (b) three key points [2] and (c) four key points [3].

Fig. 1 (a) illustrates the ROI extraction method used in [1]. It first searches for two key points in the two gaps between the forefinger and the middle finger and between the ring finger and the little finger. Then, a coordinate system can be set up according the two key points. Finally, the ROI is extracted as a constant square in this coordinate system. Figs. 1 (b) and (c) show the ROI extraction in [2] and [3]. The ideas of these methods are similar. All of them need to find some key points from the gaps between fingers. However, the palm is not a rigid object and there is no sharp corner in it, which makes the accurate locating of key points very hard. Thus, those ROI extraction methods can be used for a coarse alignment of palmprint images, and there can still be some shifting and rotation variations after ROI extraction. Although shifting the matching template [4] or using one to many matching strategy [5] can deal with some small shifting and rotation, they are time consuming and cannot really solve the rotation effects. 
To more effectively and accurately correct the shifting and rotation variations and even small scale variations between palmprint images, we propose in this paper a new alignment strategy by adapting iterative closest point (ICP) method to the palmprint principal lines. Principal lines are the most significant and stable features on the palmprint and thus they are very suitable for alignment refinement. The ICP algorithm is a classical method that was originally proposed for three dimensional (3D) shape registration [9]. It is also well suited to align two dimensional (2D) curves. In the proposed algorithm, we first extract principal lines from ROI. When matching two ROIs, we use ICP to estimate the shifting $T$, rotation $R$ and scaling $S$ between them according to the extracted principal lines. Then we use the estimated T, $R$ and $S$ to correct the ROIs so as to reduce the shifting, rotation and scale variations. The refined alignment of ROIs can bring great benefit in the subsequent palmprint verification.

The rest of this paper is organized as follows. Section 2 introduces the principal lines extraction method used in this paper. Section 3 describes the proposed ICP alignment strategy for palmprint verification. Section 4 presents the experimental results and Section 5 gives the conclusion.

\section{PRINCIPAL LINE EXTRACTION}

As shown in Fig. 2(a), principal lines are the most stable and important features in palmprint images. Fig. 2 (b) is the ROI extracted by the method illustrated in Fig. 1 (a) [1]. Although the ROI extraction reduces much the shift and rotation of palmprint images, there is still much room to further improve the palmprint alignment for higher verification accuracy. We propose in this paper a principal line based ICP method for alignment refinement. Clearly, the first step is to extract the principal lines.

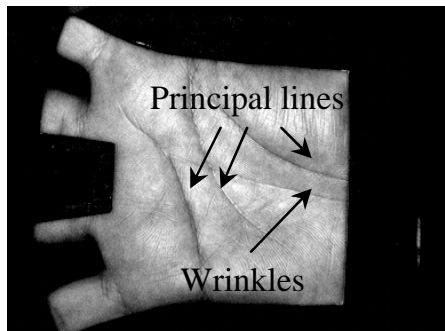

(a) Original palmprint sample

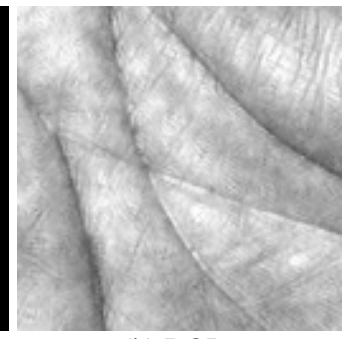

(b) ROI
Figure 2. A palmprint image and the ROI of it.

General line detection methods will extract many wrinkles and false line features from the palmprint [6]. This will introduce much difficulty to the following ICP correction. Plain and clear line features will make the ICP algorithm converge correctly and rapidly. Here we adopt Huang's palmprint principal line extraction method [7] which is based on Radon Transform (RT) [10], and then use some post-processing to enhance the results. The RT of a 2D function $f(x, y)$ is defined as

$$
R(\rho, \theta)=\int_{-\infty}^{+\infty} \int_{-\infty}^{+\infty} f(x, y) \delta(\rho-x \cos \theta-y \sin \theta) d x d y
$$

where $\rho$ is the shortest distance from the origin to the line, $\theta$ is the angle between the line and $x$-axis, and $\delta$ is a Dirac delta function which has an infinitely sharp peak response at 0 and its total integral is 1 .

A modified finite Radon transform (MFRAT) was proposed in [7] for principal line extraction:

$$
r\left[L_{k}\right]=L_{k} *(g * H-H), \quad k=1,2, \cdots, N
$$

where $H$ represents an $m \times n$ image, $g$ is a Gaussian filter, $L_{k}$ are square templates of line structures and $N$ is the total number of templates. Fig. 3 shows some example templates of size $35 \times 35$. The value of the elements is 1 on the line and 0 otherwise.

An energy-image $E$ and a direction-image $D$ are then calculated by (3) and (4) respectively

$$
\begin{aligned}
& E(i, j)=\max \left(\left|r_{i, j}\left[L_{k}\right]\right|\right), i=1,2, \cdots, m, j=1,2, \cdots, n \\
& D(i, j)=\underset{k}{\arg \max }\left(\left|r_{i, j}\left[L_{k}\right]\right|\right), i=1,2, \cdots, m, j=1,2, \cdots, n
\end{aligned}
$$

where $|\cdot|$ denotes the absolute value. With images $E$ and $D$, and by using some post-processing to remove small wrinkles, the finally thinned principal lines can be extracted. Fig. 4 illustrates the line extraction process.

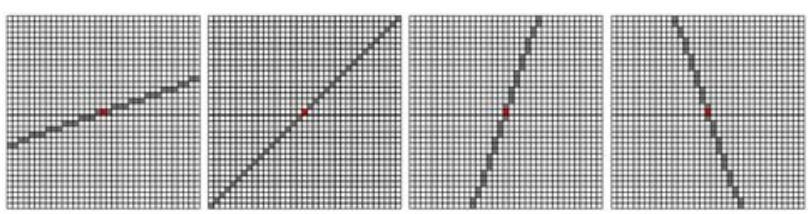

Figure 3. Some square templates used in MFRAT.

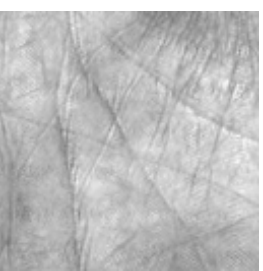

(a)

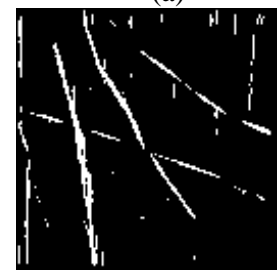

(d)

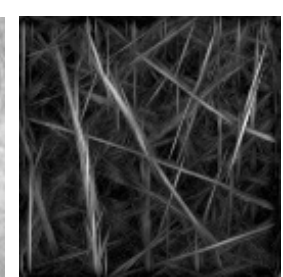

(b)

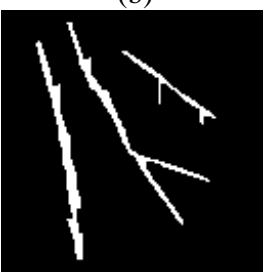

(e)

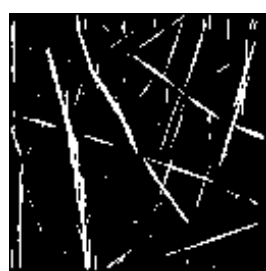

(c)

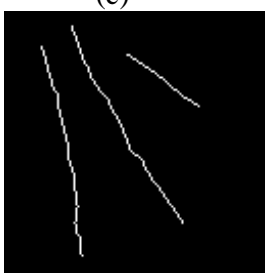

(f)
Figure 4. The extraction of principal lines. (a) Original ROI; (b) energy-image; (c) binary image of (b) after thresholding; (d) image after minor line removal of (c); (e) line map after morphological operation on (d); (f) the finally thinned principal lines. 


\section{ALIGNMENT REFINEMENT FOR PALMPRINT VERIFICATION}

With the principal lines extracted in Section 2, this section presents the principal line based ICP alignment method for palmprint verification. We first give the ICP alignment algorithm to compute the shifting, rotation and scaling between the ROI images, and then present an efficient way to combine the alignment result with the competitive code for palmprint recognition.

\subsection{Principal line based ICP alignment}

ICP algorithm was first proposed by Besl et al. for registration of 3D shapes [9]. Here, we adapted this method to estimate the shift $T$, rotation $R$ and scaling $S$ between two palmprint ROIs, and then use the estimated $T, R$ and $S$ to correct the possible shift, rotation and scale variations between them. Fig. 5 illustrates the alignment process.

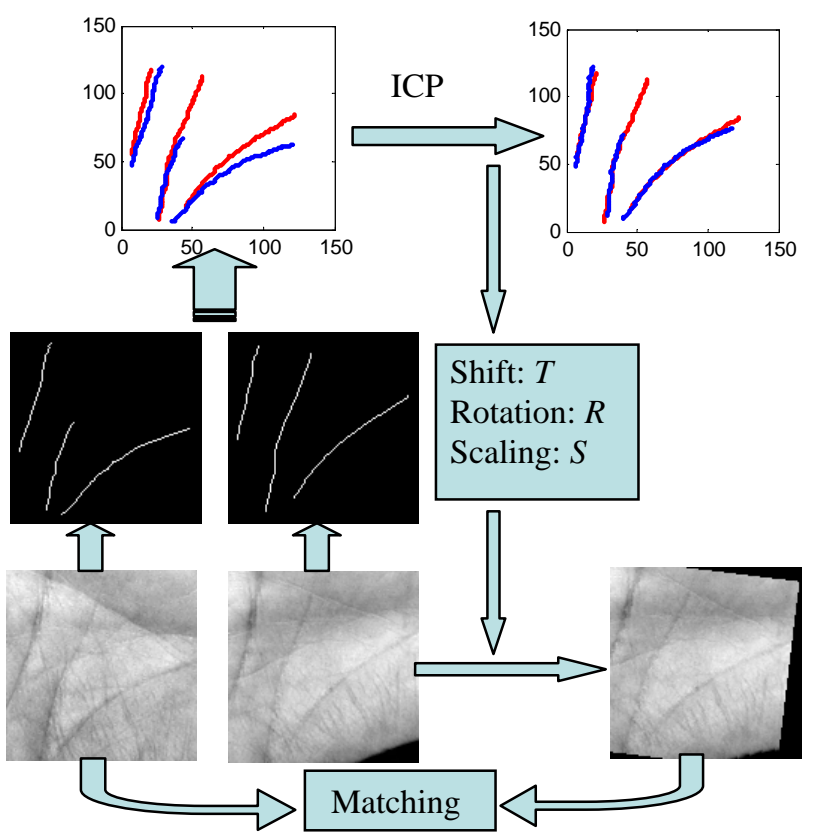

Figure 5. The principal line based ICP alignment of two ROIs.

The pseudocode of the above principal line based ICP alignment algorithm of palmprints is summarized as follows.

Algorithm of principal line based ICP alignment

\begin{tabular}{ll}
\hline Input & Two principal line point sets: $P=\left\{\vec{p}_{i}\right\}$ and \\
\hline Output & $Q=\left\{\vec{q}_{i}\right\}, i=1,2, \cdots, n$. \\
\hline Initialization & $T=\left[\begin{array}{l}0 \\
0\end{array}\right], R=\left[\begin{array}{ll}1 & 0 \\
0 & 1\end{array}\right]$, and $S=1$. \\
\hline \hline
\end{tabular}

1) For every point $\vec{p}_{i}$ in $P$, find the closest point $\vec{q}_{i}$ in $Q$ to get the corresponding point pair $\left\{\vec{p}_{i}, \vec{q}_{i}\right\}, i=1,2, \ldots, n$.

2) Calculate the total distance of corresponding

Loop point pairs: $D_{P Q}=\sum_{i=1}^{n}\left\|\vec{p}_{i}-\vec{q}_{i}\right\|_{2}$.

3) Calculate shift $T_{c}$, rotation $R_{c}$ and scaling $S_{c}$ using ICP method.

4) Update $Q=S_{c} \cdot R_{c} \cdot Q+T_{c}, T=T+T_{c}$, $R=R_{c} \cdot R$, and $S=S_{c} \cdot S$.

\begin{tabular}{ll}
$\begin{array}{l}\text { Stop } \\
\text { condition }\end{array}$ & $\begin{array}{c}D_{P Q} \text { is less than a threshold } D_{T} \text { or the iterative } \\
\text { number reaches the maximal number } N .\end{array}$ \\
\hline
\end{tabular}

\subsection{Palmprint correction and feature extraction}

Competitive code [4] is among the best algorithms for palmprint verification. It estimates the orientation (i.e. the feature) of each point in the ROI by six different oriented Gabor filters, and then codes the orientation feature for matching. More details about competitive code can be found in [4]. After obtaining the shift $T$, rotation $R$ and scaling $S$ between the ROI images by using the ICP algorithm in Section 3.1, the correction can be applied on ROI images for alignment, followed by feature extraction and matching as usual. In our test, the time whole is less than 0.5s. Such a "ROI correction first and feature extraction later" strategy has no problem in one to one (1:1) verification application. However, in one to many (1:N) identification applications, this is not a wise strategy because feature extraction will cost much time. To improve the efficiency, we can correct the feature map instead of the ROI image. In other word, we compute only once for feature extraction, and then align the extracted feature map for 1:N matching. This can save much the feature extraction time. Particularly, we ignore the scaling (because it is very small in most cases) and use shift $T$ and rotation $R$ to correct the feature map as follows.

The competitive code feature map stores the orientation of each point. For each point at $(x, y)$ in the original map, denote by $\theta$ the associated orientation. By using $T$ and $R$ to correct this position, the new orientation $\theta^{\prime}$ and position $\left(x^{\prime}, y^{\prime}\right)$ are calculated by (5) and (6) as follows:

$$
\begin{aligned}
& \theta^{\prime}=\theta+\theta_{R} \\
& {\left[\begin{array}{c}
x^{\prime} \\
y^{\prime}
\end{array}\right]=R \cdot\left[\begin{array}{l}
x \\
y
\end{array}\right]+T}
\end{aligned}
$$

where

$$
R=\left[\begin{array}{cc}
\cos \theta_{R} & -\sin \theta_{R} \\
\sin \theta_{R} & \cos \theta_{R}
\end{array}\right], \text { and } T=\left[\begin{array}{ll}
t_{x} & t_{y}
\end{array}\right] .
$$

Applying to above procedure to all the points in the original feature map, we obtain the corrected new feature map. 


\section{EXPERIMENTAL RESULTS}

The proposed approach was tested on the Hong Kong Polytechnic University (PolyU) open palmprint database which contains 7752 samples collected from 386 different palms in two sessions. The average interval between the first and second collection is 69 days. The size of the original palmprint image is $384 \times 284$ with 75 dpi resolution.

To obtain the verification accuracy of our method, each palmprint image was matched with all the other palmprint images in the database. A successful matching is called intra-class matching or genuine if the two samples are from the same class. Otherwise, the unsuccessful matching is called inter-class matching or impostor. The total number of matchings is 30,042,876. The experiments were performed on a PC with Core 2 CPU @ 2.66GHz with 2GB RAM. Fig. 6 plots the Receiver Operator Characteristic (ROC) curves of the proposed method in comparison with the original competitive code method. Table 1 lists the Equal Error Rate (EER), feature extraction time and average matching time of the above two methods. For the proposed method, the feature extraction process includes ROI extraction, principal line extraction, orientation feature extraction and coding, while the matching process includes ICP correction and feature map matching. From Fig. 6 and Table 1, we can see that the proposed method improves over $36 \%$ the EER compared with the state-of-the-art competitive code method. Although the speed of feature extraction and matching of the proposed method is slower than that of competitive code, it is fast enough for real time implementation.

Table 1. The EER, feature extraction time and average matching time by the two methods.

\begin{tabular}{|l|l|l|l|}
\hline Method & EER & Feature extraction & matching time \\
\hline$[4]$ & $0.0388 \%$ & $97 \mathrm{~ms}$ & $0.11 \mathrm{~ms}$ \\
\hline Proposed & $0.0245 \%$ & $189 \mathrm{~ms}$ & $1.76 \mathrm{~ms}$ \\
\hline
\end{tabular}

\section{CONCLUSION}

We proposed a novel alignment method for palmprint verification using ICP technique according to palmprint principal lines. It first extracts the principal lines and then uses them to estimate the shifting, rotation and scaling variations between palmprints. The estimated parameters are then used to correct the feature map for matching. The experimental results on the PolyU palmprint database (7752 samples from 386 palms) show that the proposed principal line based ICP alignment can significantly improve the palmprint recognition accuracy in real time $(<0.5 \mathrm{~s})$.

\section{ACKNOWLEDGEMENT}

The work is supported by the HK-PolyU Internal Research Grant (A-PB0B and J-BB6W) and the Hong Kong RGC General Research Fund.

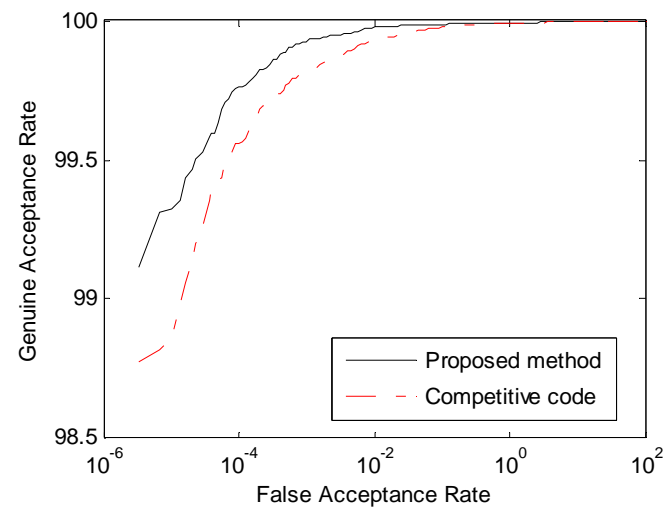

Figure 6. The ROC curves of competitive code and the proposed method.

\section{REFERENCES}

[1] D. Zhang, A.W.K. Kong, J. You, and M. Wong, "On-line palmprint identification," IEEE Transactions on Pattern Analysis and Machine Intelligence, vol. 25, no. 9, pp. 1041-1050, 2003.

[2] T. Connie, A. T. B. Jin, M. G. K. Ong, and D. N. C. Ling, "An automated palmprint recognition system," Image and Vision Computing, vol. 23, Issue 5, pp. 501-515, 2005.

[3] M. G. K. Ong, T. Connie, and T. A. B. Jin, "Touch-less palm print biometrics: Novel design and implementation," Image and Vision Computing, vol. 26, Issue 12, pp. 1551-1560, Dec. 2008.

[4] A.W.K. Kong and D. Zhang, "Competitive coding scheme for palmprint verification," Proceedings of International Conference on Pattern Recognition, vol. 1, pp. 520-523, 2004.

[5] W. Jia, D. S. Huang, and D. Zhang, "Palmprint verification based on robust line orientation code,” Pattern Recognition, vol. 41, Issue 5, pp. 1504-1513, May 2008.

[6] X.Q. Wu, D. Zhang, and K.Q. Wang, "Palm line extraction and matching for personal authentication," IEEE Transactions on Systems, Man and Cybernetics, Part A, vol. 36, Issue 5, pp. 978 987, Sept. 2006.

[7] D. S. Huang, W. Jia, and D. Zhang, "Palmprint verification based on principal lines,” Pattern Recognition, vol. 41, Issue 4, pp. 1316-1328, April 2008.

[8] L. Zhang and D. Zhang, "Characterization of Palmprints by Wavelet Signatures via Directional Context Modeling,” IEEE Transactions on Systems, Man and Cybernetics, Part B, vol. 34, no. 3, pp. 1335-1347, June 2004.

[9] P. J. Besl and N.D. McKay, "A method for registration of 3-D shapes," IEEE Transactions on Pattern Analysis and Machine Intelligence, vol. 14, no. 2, pp. 239 - 256, Feb. 1992.

[10] J. Radon, "Über die bestimmung von funktionen durch ihre integralwerte längs gewisser mannigfaltigkeiten,” Berichte Sächsische Akademie der Wissenschafter, Leipzig, Math.-Phys. Kl (69), 262-267, 1917. 